\title{
Gait and Balance Disorder in Patients with Transient Ischemic Attack or Minor Stroke
}

This article was published in the following Dove Press journal:

Neuropsychiatric Disease and Treatment

\author{
Ning Li $\mathbb{1 D}^{\prime}$ \\ Jinxin $\mathrm{Li}^{1}$ \\ Ting Gao' \\ Dandan Wang ${ }^{1,2}$ \\ Yang $\mathrm{Du}^{1,2}$ \\ Xingquan Zhao $\mathbb{D}^{1,2}$ \\ 'Department of Neurology, Beijing \\ Tiantan Hospital, Capital Medical \\ University, Beijing, People's Republic of \\ China; ${ }^{2}$ China National Clinical Research \\ Center for Neurological Diseases, \\ Beijing, People's Republic of China
}

Correspondence: Xingquan Zhao

Beijing Tiantan Hospital, Capital Medical

University, No. 119 South 4th Ring West

Road, Fengtai District, Beijing, 100070,

People's Republic of China

Tel +86-1350I03I486

Email zxq@vip.163.com
Purpose: Transient ischemic attack (TIA) and minor stroke had a high recurrence rate, resulting in potential neurological impairment. Only a few previous studies focused on gait and balance disorder in TIA and minor stroke. This study aimed to explore the relationship between gait and balance disorder and TIA/minor stroke.

Patients and Methods: Eighty-two patients with TIA/minor stroke and fifty-two healthy control participants were recruited. The gait and balance function of the two groups was tested with six measurements (Four Square Step, Tandem, Functional Reach, Gait and Pivot Turn, Timed "Up and Go", and Single Leg Balance tests). The associations between these measures and TIA/minor stroke were determined through linear and logistic regression analyses.

Results: There were no significant group differences in age, gender, body mass index, vision, and cognitive function. People with TIA/minor stroke had poorer performance in all six gait and balance measures (all $\mathrm{p}<0.05$ ). Logistic regression analysis showed TIA/minor stroke was strongly associated with gait and balance disorder (Four Square Step Test [OR, 24.07; 95\% CI 5.90-98.13; $<<0.001]$, Tandem Test [OR, 5.50; 95\% CI 1.64-18.40; $\mathrm{p}=0.006]$, Functional Reach Test [OR, 4.25; 95\% CI 1.04-17.33; p=0.044], Gait and Pivot Turn Test [OR, 3.78; 95\% CI 1.22-11.31; $\mathrm{p}=0.021$ ], Timed“Up and Go"Test [OR, 15.79; 95\% CI 2.32-107.48; $\mathrm{p}=0.005$ ], and Single Leg Balance Test [OR, 8.96; 95\% CI 2.34-34.01; $\mathrm{p}=0.001]$ ). TIA/minor stroke patients with older age, cognitive dysfunction, high K-CRP level, and severe atherosclerosis in lower limbs were more likely to have gait and balance disorder.

Conclusion: Our findings highlight the significant relationship between gait and balance disorder and TIA/minor stroke. It seems that people with TIA/minor stroke had a higher possibility to get gait and balance disorder. Gait and balance disorder following a minor stroke or TIA may be attributed to cognitive function in these patients.

Keywords: TIA, minor stroke, mobility function, intervention, cognition

\section{Introduction}

TIA and minor stroke are two of the most common cerebrovascular events with mild or transient symptoms. According to data from Chinese National Stroke Registry (CNSR), patients suffering from TIA and minor stroke accounted for $38 \%$ of inpatients with acute ischemic stroke. ${ }^{1}$ The majority of non-disabling ischemic cerebrovascular events were TIA/minor stroke. These patients had a relatively good prognosis compared to those with acute major stroke, while the recurrence rate of stroke reached $17 \%$-20\% within 90 days in TIA/minor stroke. ${ }^{2}$

After the onset of TIA/minor stroke, cerebral hemisphere cortex excitability has been shown to change on the lesion side. ${ }^{3}$ Increasing evidence has confirmed that patients with TIA/minor stroke experience persistent cognitive impairment, 
confusion, and fatigue. However, few studies in TIA/ minor stroke focus on gait and balance disorder, despite the fact that it may interfere with daily activities and reduce quality of life. Gait and balance disorder is the most important determinant of independent living in the elderly, which can result in falling, fracture, and consequent disability, even death. ${ }^{4}$ It may occur before or concomitantly with the disease but is easily overlooked in milder diseases. Therefore, this study tested the hypothesis that gait and balance function is decreased in TIA/minor stroke patients compared to the healthy controls, even though the patients had no previous walking problems or decreased muscle strength.

\section{Patients and Methods}

\section{Study Design}

We designed an observational study. This study invited TIA/minor stroke patients from inpatient ward at Beijing Tiantan Hospital from November 2018 to October 2019. All gait and balance tests were performed by trained researchers blinded to participants within 2 weeks of the onset of minor stroke or the last attack of TIA. The order of testing was consistent for each participant and performed only once. The study was approved by the Ethics Committees of Beijing Tiantan Hospital and was performed in accordance with the guidelines of the Helsinki Declaration. Written informed consent was obtained from all participants.

\section{Participants Selection}

Inclusion criteria were: (1) age $\geq 45$ years; (2) diagnosis of acute TIA or minor stroke by a professional neurologist; (3) no reported falls in the year before TIA/minor stroke; and (4) ability to sign informed consent.

Exclusion criteria were: (1) current or past history of bone or joint diseases, sequelae of stroke, Parkinson's disease, postural hypotension, and other diseases that can affect gait or balance function; (2) taking medication that includes drugs known to affect gait balance, such as dopaminergic, antiepileptic drugs, etc; and (3) diagnosis of mental illness or depression.

A group of participants without TIA/minor stroke but meeting other inclusion/exclusion criteria were also recruited as controls from the medical examination clinic at the same time. TIA was defined as a transient episode of neurological dysfunction caused by focal brain, spinal cord, or retinal ischemia, without acute infarction or tissue injury on magnetic resonance imaging (DWI). ${ }^{2}$ Acute minor stroke was defined by a score of 3 or less on the National Institutes of Health Stroke Scale (NIHSS) and could not include muscle strength score. ${ }^{5}$

\section{Data Sources and Measurement Demographic and Clinical Characteristics}

In each assessment, participants were interviewed by questionnaires that ascertained age, sex, height, weight (calculated BMI), years of education, medications, and health conditions. Clinical characteristics were recorded including vision (superior and inferior vision), $\mathrm{ABCD}^{2}$ score/ NIHSS score, localization signs (anterior or posterior circulation), and localization of MRI lesion (lobe, paraventricular, basal ganglia, thalamus, brainstem, cerebellum).

Mini-Mental State Examination (MMSE) and Montreal Cognitive Assessment (MOCA): tests of cognitive function.

Hospital Anxiety and Depression Scale (HADS): a 14item self-report questionnaire for emotional changes in daily life and behavior in the past month. ${ }^{6}$

Five Times Sit-to-Stand Test (FTSST): a test assessing lower limb muscle strength. ${ }^{7}$

\section{Gait and Balance Measures}

Timed“Up and Go"Test (TUG): the time required to walk 3 meters forward and back, a test of gait and dynamic balance. $^{8}$

Four Square Step Test (FSST): the time required to step clockwise in four directions and then step back anticlockwise, a test of gait and dynamic balance ability. ${ }^{9}$

Functional Reach Test (FRT): the maximum distance of distal forearm extension of both upper limbs when both lower limbs are fixed, a static balance test. ${ }^{10}$

Gait and Pivot Turn (GPT): the time required to turn around quickly after hearing instructions, derived from the Functional Gait Assessment scale, a test of dynamic balance ability. ${ }^{11}$

Single Leg Balance Test (SLBT): the amount of time one is able to stand balanced on one leg, a static balance test. $^{12}$

Tandem Test: the amount of time one is able to stand in a line with both feet and keep balance, a static balance test derived from the Berg scale. ${ }^{13}$

These six standardized, objective, and validated tests were used to assess mobility function in all participants, and include both quantitative data (TUG, FSST, FRT, and GPT) and qualitative data (SLBT, Tandem). The presence 
of gait and balance disorder was defined as: scoring 1 standard deviation below (or above) the mean of the control group for TUG, FSST, GPT, and FRT, or scores less than 4 for SLBT and Tandem. ${ }^{14}$

\section{Sample Size}

The sample size was not predetermined for this pilot study.

\section{Statistical Analysis}

Continuous data are reported as mean \pm standard deviation (SD) for normally distributed data, median (interquartile range) for non-normally distributed data, and categorical data are reported as numbers and percentages (\%). All analyses were undertaken using SPSS version 22.0 (IBM Corporation). Baseline differences in characteristics between the groups were analyzed using $\mathrm{T}$ tests, Wilcoxon signed-rank tests, and $\chi^{2}$ tests. Between-group differences in the frequency of gait and balance disorders between groups was estimated with $\chi^{2}$ and logistic regression tests. Spearman correlation and Kendall's tau-b correlation analysis were used to assess relationships between selected characteristics and gait and balance measurements in TIA/minor stroke patients. Multivariate regression analysis was used to further determine associated factors of gait and balance function in TIA/minor stroke patients. $P$ values $\leq 0.05$ indicated statistical significance.

\section{Results}

According to the inclusion/exclusion criteria, 82 participants with TIA/minor stroke out of the total 256 screened were eligible and agreed to participate (16 TIA and 66 minor stroke), 52 participants out of 66 screened were included in the control group. The median time from symptom onset to gait and balance function assessment was 13 days.

Compared to controls, patients with TIA/minor stroke had a higher number of drinking and, smoking, and tend to use $\geq 4$ kinds of medications. Other basic characteristics such as age, gender, years of education, medical history of hypertension and diabetes, vision, MMSE and MOCA score, HADS score, and FTSST showed no significant differences between groups (Table 1). For minor stroke patients, the median NIHSS score at presentation was 1 (0-2). As high as $63.4 \%$ of the infarction lesions were located in the anterior circulation, with basal ganglia as the most $(23.2 \%)$, followed by frontal lobe $(12.5 \%)$ and paraventricular $(8.5 \%)$. For TIA patients, the median $\mathrm{ABCD}^{2}$ score was 4.5 (2.5-5.0).

\section{Gait and Balance Measurements Outcomes}

Patients with TIA/minor stroke had significantly poorer performance on all measures of gait and balance function (Table 2, Figure 1-2). After defining gait and balance disorder with the above measurements, proportions of patients with the disorder remained higher in TIA/minor stroke group compared to controls. When gait and balance disorder was defined according to the FSST, the difference between groups was the largest $(64.2 \%$ vs $7.7 \%, p=0.011)$. Similar results were obtained when the disorder was defined based on the other measurements (TUG, 30.5\% vs $11.5 \%$; FRT, $25.9 \%$ vs $9.6 \%$; GPT, $42.7 \%$ vs $15.7 \%$; SLBT, $46.3 \%$ vs $13.5 \%$; Tandem, $40.2 \%$ vs $17 . \%$; respectively, all $\mathrm{p}<0.05)$. In multivariate-adjusted logistic regression, TIA/minor stroke was independently associated with gait and balance disorder; the association remained after adjusting the relevant factors with ORs ranging from 4.25 to 24.07 (Table 3, Figure 3).

\section{Risk Factors on Gait and Balance in TIA/ Minor Stroke}

We next assessed the relationship between each gait and balance measurement and selected demographic and clinical characteristics in TIA/minor stroke patients. Age, sex, cognitive function (scores on MMSE and MOCA), use $\geq 4$ medications, presence of $\geq 3$ combined diseases, vision, lower limb strength (FTSST), atherosclerosis in lower limbs, and K-CRP were all correlated with one or more gait and balance measurements. Among these variables, age (age-TUG, $\mathrm{r}=0.490, \mathrm{p}<0.001$; age-FRT, $\mathrm{r}=-0.380, \mathrm{p}<0.001$; ageSLBT, $\quad \mathrm{r}=-0.440, \quad \mathrm{p}<0.001 ;$ age-Tandem, $\mathrm{r}=0.019$, $\mathrm{p}=0.019$ ) and MMSE (MMSE-TUG, $\mathrm{r}=-0.354$, $\mathrm{p}=0.002$; MMSE-GPT, $\mathrm{r}=-0.239, \mathrm{p}=0.039$; MMSESLBT, $\mathrm{r}=0.218, \mathrm{p}=0.023$; MMSE-Tandem, $\mathrm{r}=0.199$, $p=0.049$ ) had the most significant correlation coefficients with 4 pairs, followed by FTSST and inferior vision (Table 4). Location of the MRI lesion, BMI, HADS, vascular risk factors (smoking, drinking, hypertension, diabetes), and levels of other laboratory indices (leucocyte, hemoglobin, D-dimer, glycated hemoglobin, low-density lipoprotein, high-density lipoprotein, very low-density lipoprotein, homocysteine) were not statistically correlated with gait and balance measurements (shown in supplemental Tables S1-S4). As for the six gait and balance measurements, the 
Table I Demographic and Clinical Characteristics of Participants

\begin{tabular}{|l|l|l|l|}
\hline & TIA/Minor Stroke (n=82) & Controls (n=52) & P value \\
\hline Age(years) & $62.0(55.8-67.3)$ & $61.0(56.0-66.5)$ & 0.515 \\
Female (\% yes) & $21(25.6 \%)$ & $19(36.5 \%)$ & 0.178 \\
BMI(kg/m²) & $25.2(22.8-27.7)$ & $24.0(22.4-26.2)$ & 0.092 \\
Education (years) & $9(9-12)$ & $12(9-15)$ & 0.182 \\
Smoking (\% yes) & $48(58.5 \%)$ & $17(32.7 \%)$ & 0.004 \\
Drinking (\% yes) & $41(50.0 \%)$ & $11(21.2 \%)$ & 0.001 \\
Hypertension (\% yes) & $54(65.9 \%)$ & $27(51.9 \%)$ & 0.108 \\
Diabetes (\% yes) & $30(36.6 \%)$ & $14(26.9 \%)$ & 0.246 \\
Combined disease $\geq 3$ kinds (\% yes) & $23(28.0 \%)$ & $19(36.5 \%)$ & 0.302 \\
Medication use $\geq 4$ kinds (\% yes) & $18(22.0 \%)$ & $19(38.8 \%)$ & 0.038 \\
Psychotropic drugs use (\% yes) & $4(4.9 \%)$ & $1(1.9 \%)$ & 0.379 \\
Superior vision & $0.6(0.5-0.8)$ & $0.6(0.5-0.8)$ & 0.954 \\
Inferior vision & $0.5(0.4-0.6)$ & $0.6(0.4-0.8)$ & 0.501 \\
MMSE (score) & $28.0(27.0-30.0)$ & $28.0(27.0-29.0)$ & 0.362 \\
MoCA (score) & $23.5(19.5-26.0)$ & $23(20.0-24.5)$ & 0.289 \\
HADS (score) & $4.0(3.0-7.0)$ & $4.5(2.0-7.5)$ & $13.9(12.0-15.8)$ \\
FTSST(s) & $13.0(11.5-14.4)$ & 0.858 \\
\hline
\end{tabular}

Notes: Values are presented as median (IQR) or percentages. Percentages are based on total number of individuals for each group.

Abbreviations: MMSE, Mini-Mental State Examination; MoCA, Montreal Cognitive Assessment; HADS, Montreal Cognitive Assessment; FTSST, Five Times Sit-to-Stand Test.

Table 2 Results of Six Gait and Balance Measurements in Two Groups

\begin{tabular}{|c|c|c|c|}
\hline & $\begin{array}{l}\text { TIA/Minor Stroke } \\
(\mathrm{n}=\mathbf{8 2})\end{array}$ & $\begin{array}{l}\text { Controls } \\
(n=52)\end{array}$ & p value \\
\hline $\operatorname{TUG}(s)^{\mathrm{a}}$ & $11.7 \pm 2.0$ & $10.5 \pm 1.9$ & 0.001 \\
\hline FSST $(s)^{a}$ & $12.7 \pm 2.8$ & $9.4 \pm 2.3$ & $<0.001$ \\
\hline GPT $(s)^{a}$ & $2.6 \pm 0.5$ & $2.2 \pm 0.6$ & $<0.001$ \\
\hline FRT $(\mathrm{cm})^{\mathrm{b}}$ & $27.8 \pm 6.1$ & $32.2 \pm 6.5$ & $<0.001$ \\
\hline SLBT (Score) ${ }^{b}$ & & & $<0.001$ \\
\hline I & $10(12.2 \%)$ & $0(0.0 \%)$ & \\
\hline 2 & 8 (9.8\%) & $0(0.0 \%)$ & \\
\hline 3 & 20 (24.4\%) & 7 (I3.5\%) & \\
\hline 4 & 44 (53.7\%) & 45 (86.5\%) & \\
\hline Tandem (Score) & & & 0.005 \\
\hline 1 & $0(0.0 \%)$ & $0(0.0 \%)$ & \\
\hline 2 & $2(2.4 \%)$ & $0(0.0 \%)$ & \\
\hline 3 & 31 (37.8\%) & $9(17.3 \%)$ & \\
\hline 4 & 49 (59.8\%) & 43 (82.7\%) & \\
\hline
\end{tabular}

Notes: ${ }^{a}$ Higher number means worse performance; ${ }^{b}$ higher score means better performance.

Abbreviations: TUG, Timed "Up and Go" Test; FSST, Four Square Step Test; FRT, Functional Reach Test; GPT, gait and pivot turn; SLBT, Single Leg Balance Test.

significant correlation was most prevalent between the Tandem test and corresponding variables were the most, followed by the SLBT, and the TUG test. We chose the Tandem and TUG test, one for categorical variables and the other for continuous variables, for further multivariate regression analysis.
For TIA/minor stroke patients, age and K-CRP were independently associated with Tandem score. Statistically, patients with severe lower limb stenosis were 0.0002 times more likely to have a better balance performance than those without stenosis $(95 \% \mathrm{CI}, 0.00,0.97 ; \mathrm{p}=0.049$ ), and 0.001 times more likely than those with mild to moderate stenosis (OR, 0.0001; 95\% CI, 0.00, 0.47; $\mathrm{p}=0.032$ ). In the linear regression of the TUG test, TUG test time dropped by 0.57 seconds for each point added to MMSE score (95\% CI: $-0.73,-0.06 ; \mathrm{p}=0.023$ ) (Table 5).

\section{Discussion}

In our study, we found that patients with TIA/minor stroke experienced gait and balance disorder after the disease at a higher rate than controls without TIA/minor stroke. This disorder may be attributed to cognitive impairment, aging, and atherosclerotic processes. To the best of our knowledge, this is the first pilot study to demonstrate the relationship between TIA/minor stroke and gait and balance function in China.

Clinical managements after TIA/minor stroke were focused on stroke prevention; however, previous studies have suggested that patients may experience ongoing cortical dysfunction. Halevi found that TIA patients have cognitive impairment within $24 \mathrm{~h}$ of onset. ${ }^{15}$ Moran reported that TIA/ minor stroke patients may experience residual impairments including fatigue, anxiety, depression, post-traumatic stress disorder (PTSD), and cognitive impairment for which they 
A

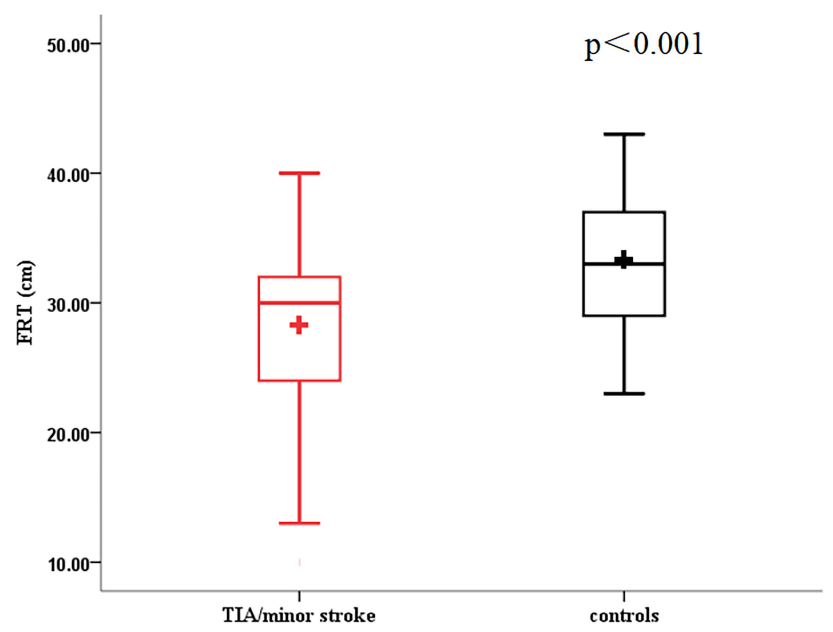

C

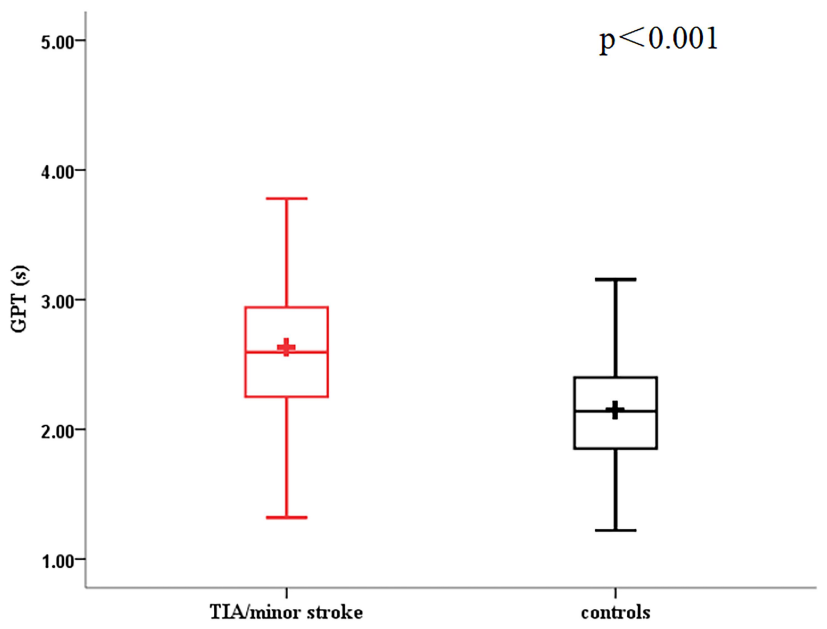

B

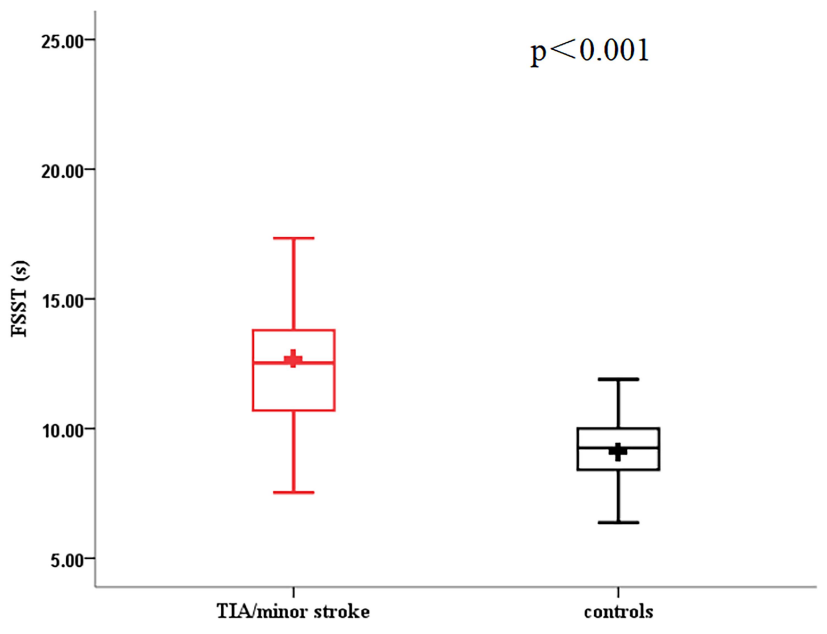

D

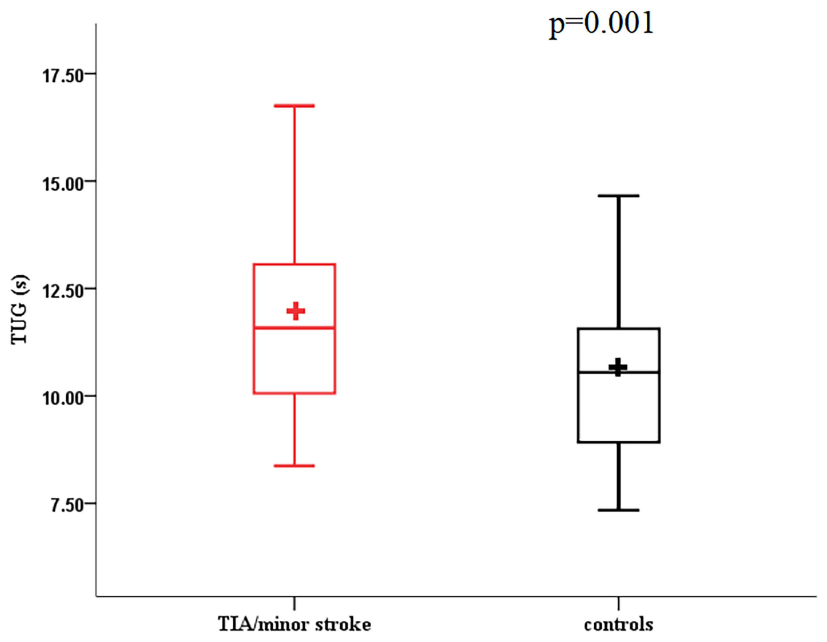

Figure I Boxplot (A-D) showed the distribution of the gait and balance measures for the four continuous variables. TUG, Timed "Up and Go" Test; FSST, Four Square Step Test; GPT, gait and pivot turn; FRT, Functional Reach Test.

are not routinely offered treatment. ${ }^{16}$ Therefore, in our study, because gait and balance are also related to proper functioning of higher cerebral cortical structures, we further demonstrated a significant relationship between gait and balance function and TIA/minor stroke. In previous studies, Israelsson studied 76 patients with TIA and found 16\% of them showed observed disturbance in the gait analysis. ${ }^{17}$ Batchelor found that people with TIA/minor stroke had worse performance on mobility and cognition tests. ${ }^{18}$ But their sample may have represented those with relatively high functional status given that their inclusion criteria did not include severe restrictions on muscle strength condition. In our study, participants with decreased muscle strength were excluded, and there was no significant group difference on the lower limb muscle strength test (FTSST). We believe that the sample of our study is more representative and thus the conclusion is more convincing.

In order to find rational explanations for the result, we analyzed potential causative factors and raised several possibilities. Firstly, in our study, we found that the cognitive function was significantly related to gait and balance disorder. The patients performed worse especially under more cognitively challenging conditions (FSST, TUG). The balance reflex is achieved through autonomic posture adjustment and feed-forward posture adjustment. ${ }^{19-21}$ It requires the coordination of visual, vestibular, proprioceptive and, in particular, cognitive integration capabilities. ${ }^{22}$ An observational study of elderly participants in Spain showed a significant correlation between cognition and frailty. ${ }^{23}$ During the aging process, coordination among the systems 


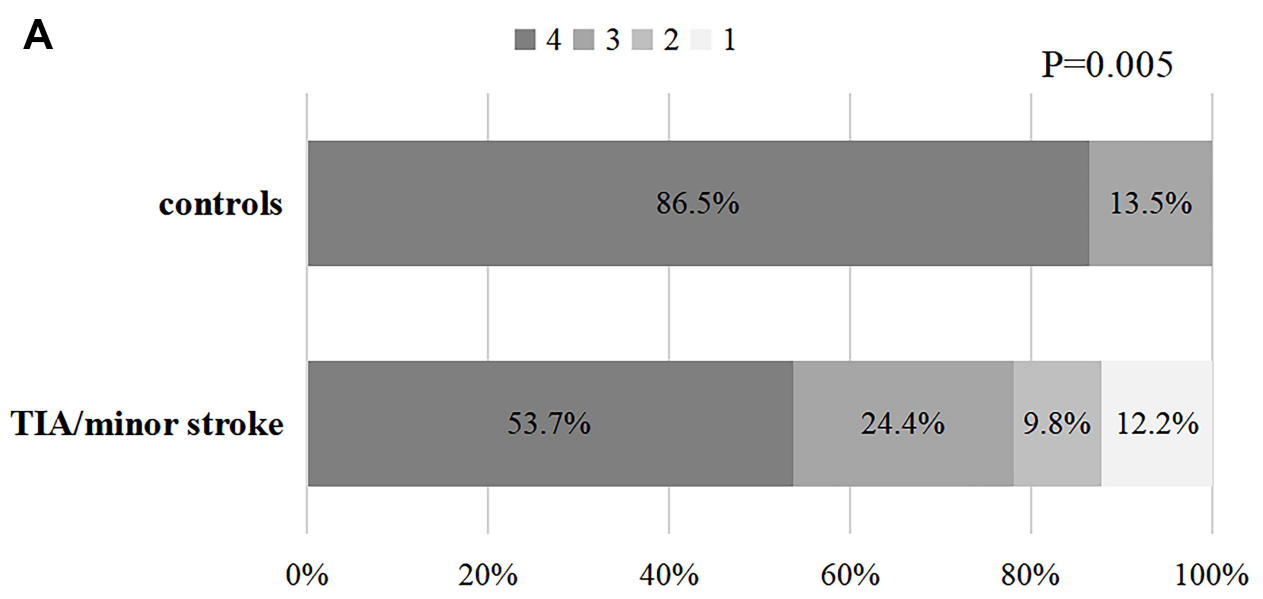

SLBT Scale Propotion

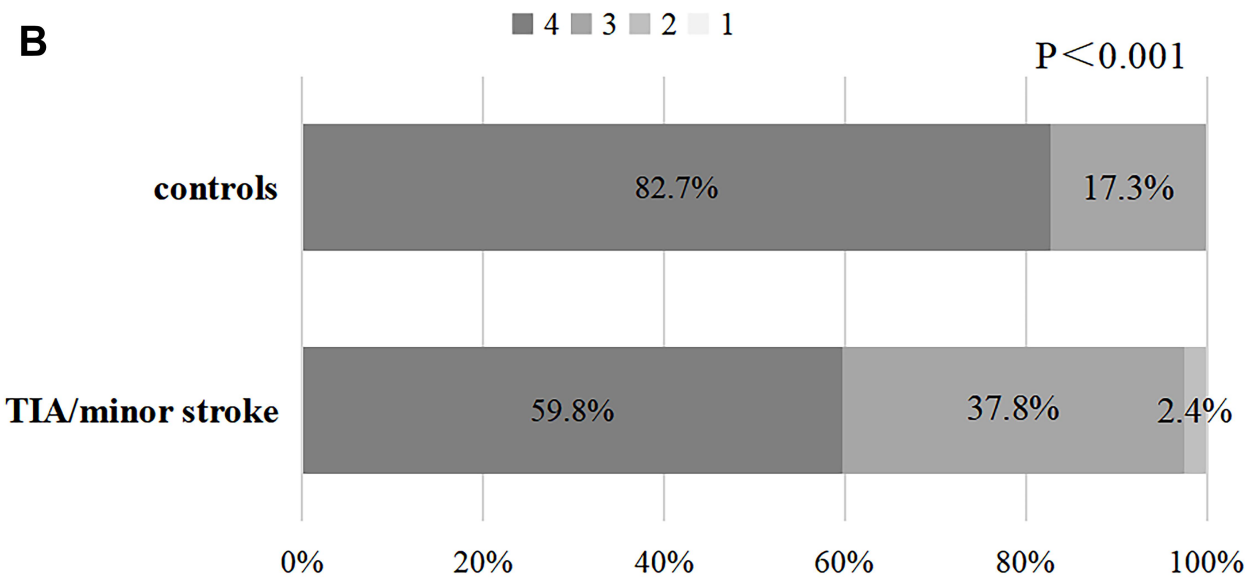

Tandem Scale Propotion

Figure 2 Percentage bar chart (A) and (B) showed the score distribution of the gait and balance measures for the two categorical variables. SLBT, Single Leg Balance Test.

declines, and the original balance control process based on body reflexes relies more on cognitive function. ${ }^{24}$ Decreased cognitive integration function may lead to deviations in judgment of the surrounding environment. The cognitive integration function of the prefrontal cortex may not be coordinated with the executive function of the body, which leads to impaired mobility function. ${ }^{24,25}$

Secondly, our study found that patients with severe atherosclerosis in lower limbs or high K-CRP level have a higher probability of having gait and balance disorder. Gray reported that asymptomatic carotid artery stenosis is associated with impaired mobility, and the impairment increased with worsening stenosis severity. ${ }^{14}$ Atherosclerosis was also associated with microembolism and asymptomatic infarction, which have been reported to lead to decreased mobility. ${ }^{26}$ As atherosclerosis and the related active inflammation cause neurodegeneration, that may be an essential causative factor contributing to gait and balance disorder. ${ }^{27}$

In addition, chronic hypoperfusion and abnormal cerebrovascular hemodynamics in the TIA/minor stroke population lead to the loss of neurons and neurotrophic dysfunction, ${ }^{28-30}$ which destroys the neurological pathways related to motor control, and subsequently lead to abnormal walking. Israelsson also reported that TIA patients with increased white matter lesions (WML) coped worse with the most advanced balance tests (single 
Table 3 Effects of TIA/Minor Stroke on Gait and Balance Disorder

\begin{tabular}{|l|l|l|l|l|l|l|}
\hline & \multicolumn{2}{|l|}{ TIA/Minor Stroke } & \multirow{2}{*}{ Controls } & \multicolumn{2}{l|}{ Crude } & \multicolumn{2}{l|}{ Adjusted } \\
\cline { 4 - 7 } & & & OR(95\% CI) & p value & \multicolumn{2}{l|}{ OR(95\% CI) } \\
\hline TUG (s) & $25(30.5 \%)$ & $6(11.5 \%)$ & $3.36(1.27-8.89)$ & 0.014 & $15.79(2.32-107.48)$ & 0.005 \\
FSST (s) & $52(64.2 \%)$ & $4(7.7 \%)$ & $21.52(7.05-65.72)$ & $<0.00 I$ & $24.07(5.90-98.13)$ & $<0.001$ \\
GPT (s) & $35(42.7 \%)$ & $8(15.7 \%)$ & $4.00(1.67-9.58)$ & 0.002 & $3.78(1.22-11.31)$ & 0.021 \\
FRT (cm) & $21(25.9 \%)$ & $5(9.6 \%)$ & $3.29(1.15-9.38)$ & 0.026 & $4.25(1.04-17.33)$ & 0.044 \\
SLBT (score) & $38(46.3 \%)$ & $7(13.5 \%)$ & $9.02(2.84-28.70)$ & $<0.001$ & $8.96(2.34-34.01)$ & 0.001 \\
Tandem (score) & $33(40.2 \%)$ & $9(17.3 \%)$ & $3.22(1.39-7.48)$ & 0.007 & $5.50(1.64-18.40)$ & 0.006 \\
\hline
\end{tabular}

Notes: The disorder is defined as: I standard deviation below (or above) the mean of the control group (TUG, FSST, GPT, FRT) or scores less than 4 (SLBT, Tandem). $P$ value adjusted for: age, gender, BMI, smoking, drinking, combined medication $\geq 4$ kinds, MMSE, MoCA, FTSST.

leg stance and Tandem test). ${ }^{17}$ Moreover, decreased cerebral perfusion may be related to impaired cognitive function, which can indirectly affect gait and balance. ${ }^{31}$ Further studies including cerebral perfusion imaging and functional scanning are needed as these were not performed in our study population. Thus, it may be a combination of mechanisms, including cognitive dysfunction, atherosclerosis and related inflammation, hypoperfusion, and abnormal cerebrovascular hemodynamic that contribute to impaired mobility in TIA/minor stroke.

Motor function decreased with increasing age and vascular risk factors. Previous studies suggested that a single disease may not be significantly related to gait balance disorder, but when more than three basic diseases were combined as a factor, it was obviously related, and the more basic diseases, the greater the risk ratio. ${ }^{22}$ Navarro-Flores recently found that using more than 5 different medications on a regular basis is correlated with the fragility in older participants, which may increase the risk of falls. ${ }^{23}$ Findings by Bloch and Woolcott JC

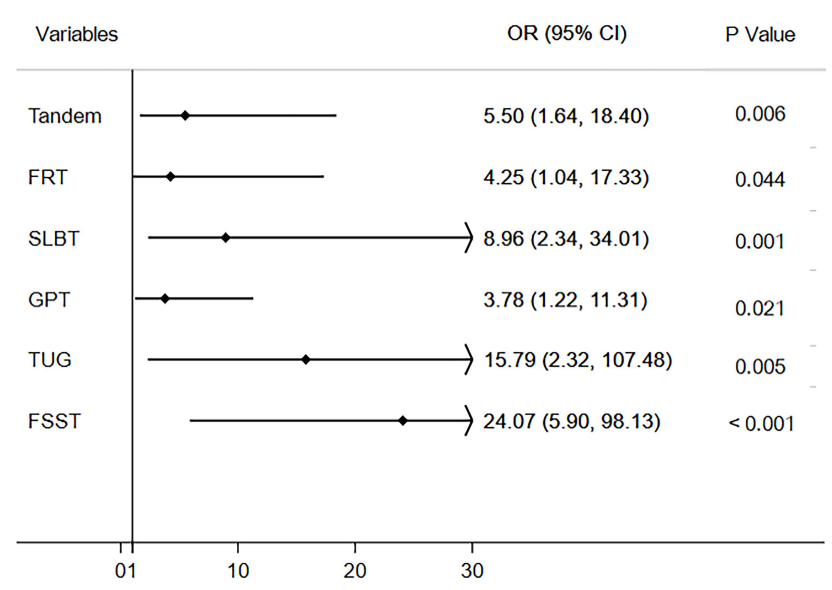

Figure 3 Adjusted odds ratios of presenting TIA/minor stroke (independent variable) on gait and balance dysfunction (dependent variable). both showed that gait and balance function may be influenced by combined use of multiple drugs, especially psychotropic medication. ${ }^{32,33}$ Good control of vascular risk factors and rational prescription of medications remains important to patient health and prognosis. Clinical controlled trials have found that effective single interventions included improving the safety of the living environment, exercising and physical therapy, improving vision, and reducing medication. ${ }^{34,35}$ Studies using automated mechanical peripheral stimulation, like plantar stimulation, have been shown to improve postural instability in individuals with Parkinson's disease. ${ }^{36}$ The most effective strategy may be to intervene with multiple risk factors. ${ }^{22,37,38}$ A management strategy employing dual-task cognitive exercise training and active cerebrovascular risk factor management may improve gait and balance function and reduce the risk of falls. ${ }^{39}$

This project had important clinical implications. The immediate meaning is that clinicians can identify patients with a higher tendency to have gait and balance disorder earlier, manage intervention and rehabilitation treatment in a timely manner, and improve prognosis. Regarding stroke prevention, $\mathrm{ABCD}^{2}$ and ESSEN scales are commonly used to predict the risk of stroke and guide the selection of treatment in TIA/minor stroke patients. However, these tools mainly use baseline information, such as age, symptom, and medical history, and rarely include clinical objective indicators. ${ }^{40-42}$ Further studies may embrace the idea that gait and balance tests may be efficient clinical indicators to enrich the set of evaluation tools used for secondary prevention of stroke.

Our study may have some potential limitations. First, all participants were recruited from one hospital, which may introduce selection bias. We will try to develop further multicenter studies to better understand the mechanisms involved and to determine effective interventions. Second, we only tested the baseline gait and balance function in our study, 
Table 4 Correlation Analysis Between Clinical Characteristics and Gait and Balance Function in TIA/Minor Stroke

\begin{tabular}{|c|c|c|c|c|c|c|c|}
\hline & & Tandem & TUG & FSST & FRT & GPT & SLBT \\
\hline \multirow[t]{2}{*}{ Age (years) } & $r$ & -0.258 & 0.490 & 0.138 & -0.380 & 0.154 & -0.440 \\
\hline & $\mathrm{P}$ & 0.019 & $<0.001$ & 0.221 & $<0.001$ & 0.168 & $<0.00$ \\
\hline \multirow[t]{2}{*}{ Female } & $r$ & -0.205 & 0.127 & -0.076 & -0.116 & 0.009 & -0.261 \\
\hline & $\mathrm{P}$ & 0.063 & 0.164 & 0.408 & 0.224 & 0.920 & 0.012 \\
\hline \multirow[t]{2}{*}{ Combined disease $\geq 3$ kinds } & $r$ & -0.261 & -0.103 & 0.013 & 0.121 & 0.156 & 0.165 \\
\hline & $\mathrm{P}$ & 0.018 & 0.258 & 0.890 & 0.205 & 0.088 & 0.112 \\
\hline \multirow[t]{2}{*}{ Medication use $\geq 4$ kinds } & $r$ & -0.229 & -0.173 & -0.052 & 0.055 & 0.097 & 0.096 \\
\hline & $\mathrm{P}$ & 0.037 & 0.058 & 0.574 & 0.563 & 0.292 & 0.356 \\
\hline \multirow[t]{2}{*}{ Superior vision } & $r$ & 0.261 & -0.225 & -0.150 & 0.090 & 0.021 & 0.268 \\
\hline & $\mathrm{P}$ & 0.022 & 0.049 & 0.196 & 0.439 & 0.855 & 0.018 \\
\hline \multirow[t]{2}{*}{ Inferior vision } & $r$ & 0.370 & -.0215 & -0.160 & 0.147 & -0.002 & 0.237 \\
\hline & $\mathrm{P}$ & 0.001 & 0.061 & 0.167 & 0.206 & 0.987 & 0.038 \\
\hline \multirow[t]{2}{*}{ MMSE (score) } & $r$ & 0.199 & -0.354 & -0.169 & 0.211 & -0.239 & 0.218 \\
\hline & $\mathrm{P}$ & 0.049 & 0.002 & 0.147 & 0.069 & 0.039 & 0.023 \\
\hline \multirow[t]{2}{*}{ MoCA (score) } & $r$ & 0.078 & -0.264 & -0.245 & 0.058 & 0.018 & 0.115 \\
\hline & $\mathrm{P}$ & 0.447 & 0.029 & 0.044 & 0.638 & 0.882 & 0.239 \\
\hline \multirow[t]{2}{*}{ FTSST(s) } & $r$ & -0.095 & 0.580 & 0.461 & -0.207 & 0.259 & -0.127 \\
\hline & $\mathrm{P}$ & 0.293 & $<0.001$ & $<0.001$ & 0.064 & 0.019 & 0.140 \\
\hline \multirow[t]{2}{*}{ Intravenous thrombolysis } & $r$ & -0.245 & -0.019 & 0.029 & -0.183 & -0.214 & 0.357 \\
\hline & $\mathrm{P}$ & 0.028 & 0.867 & 0.800 & 0.106 & 0.057 & 0.001 \\
\hline \multirow[t]{2}{*}{$\mathrm{K}-\mathrm{CRP}(\mathrm{mg} / \mathrm{L})$} & $r$ & -0.212 & 0.038 & 0.401 & -0.168 & 0.045 & -0.061 \\
\hline & $\mathrm{P}$ & 0.222 & 0.830 & 0.017 & 0.335 & 0.797 & 0.728 \\
\hline \multirow[t]{2}{*}{ Atherosclerosis in lower limbs } & $r$ & -0.128 & -0.098 & -0.031 & -0.251 & 0.048 & -0.133 \\
\hline & $\mathrm{P}$ & 0.298 & 0.428 & 0.802 & $0.04 I$ & 0.697 & 0.278 \\
\hline
\end{tabular}

Notes: Only variables with statistically significance were shown; boldfaced $p$ values indicate significant findings $(p<0.05)$; atherosclerosis in lower limbs was categorized as: Normal, Stenosis, Occlusion.

Table 5 Multivariate-Adjusted Odds Ratios and 95\% Confidence Intervals of Tandem and TUG Test in TIA/Minor Stroke

\begin{tabular}{|c|c|c|c|c|}
\hline & Tandem $^{a}$ & & TUG $^{\mathbf{b}}$ & \\
\hline & OR $(95 \% \mathrm{CI})$ & $\mathbf{p}$ & $\beta(95 \% \mathrm{Cl})$ & $\mathbf{p}$ \\
\hline Age(years) & $0.63(0.4 I-0.87)$ & 0.041 & $0.04(-0.07-0.14)$ & 0.477 \\
\hline Female & $7.60(0.51-269.62)$ & 0.266 & $0.03(-2.04-2.09)$ & 0.979 \\
\hline Medication use $\geq 4$ kinds & $0.86(0.04-16.96)$ & 0.921 & $1.77(-0.19-3.73)$ & 0.074 \\
\hline Inferior vision & $10.30(0.01-3333.03)$ & 0.524 & $-1.33(-4.64-1.99)$ & 0.409 \\
\hline FTSST (s) & $3.01(0.98-9.22)$ & 0.054 & $0.31(-0.04-0.65)$ & 0.078 \\
\hline MMSE Score & $0.50(0.20-1.23)$ & 0.131 & $-0.57(-0.73--0.06)$ & 0.023 \\
\hline $\mathrm{K}-\mathrm{CRP}(\mathrm{mg} / \mathrm{L})$ & $0.54(0.30-0.89)$ & 0.046 & $-0.07(-0.32-0.18)$ & 0.575 \\
\hline Intravenous thrombolysis & $0.23(0.05-1.03)$ & 0.055 & $0.08(-1.33-2.22)$ & 0.605 \\
\hline Atherosclerosis in lower limbs & & & $-0.62(-1.90-0.65)$ & 0.078 \\
\hline Normal-Stenosis & $0.57(0.04-8.86)$ & 0.684 & & \\
\hline Normals-Occlusion & $0.0002(0.00-0.97)$ & 0.049 & & \\
\hline Stenosis-Occlusion & $0.000 \mathrm{I}(0.00-0.47)$ & 0.032 & & \\
\hline
\end{tabular}

Notes: ${ }^{\circ}$ Ordinal regression was used in Tandem test; blinear regression was used in TUG test. P value adjusted for: age, female, medication use $\geq 4$ kinds, Inferior vision, FTSST, MMSE score, K-CRP, intravenous thrombolysis, atherosclerosis in lower limbs. 
long time follow-up tests need to be performed and compared. We will continue to focus on the patients' stroke recurrence events to further explore the impact of gait and balance disorder on stroke recurrence. Future research should investigate mechanistic associations between TIA/minor stroke and mobility impairments, including the combination of advanced electric gait recorder and cerebral perfusion imaging. Furthermore, additional research should develop cost-effective measures to identify TIA/minor stroke patients with gait and balance impairment and improve the healthcare and rehabilitation of these patients.

\section{Conclusion}

TIA/minor stroke was independently associated with gait and balance disorder. Age, cognitive function, and atherosclerosis burden were correlated with the gait and balance function of TIA/minor stroke patients. These results suggest that TIA/ minor stroke is not only a "non-disabling" disease but that there are many potential dysfunctions to be discovered. The exploration of associated mechanisms and early identification and management of risk factors may delay the progression. Although there are challenges and obstacles, gait and balance assessment strategies should be incorporated into the clinical practice of TIA/minor stroke.

\section{Acknowledgments}

This study was supported by National Science and Technology Major Project (2017ZX09304018). Authors would like to express sincere gratitude to all the participants. And thanks EditSprings for the expert linguistic services provided.

\section{Disclosure}

The authors report no conflicts of interest in this work.

\section{References}

1. Wang P, Wang Y, Zhao X, et al. In-hospital medical complications associated with stroke recurrence after initial ischemic stroke: a prospective cohort study from the China National Stroke Registry. Medicine. 2016;95(37):e4929. doi:10.1097/MD.0000000000004929

2. Easton JD, Saver JL, Albers GW, et al. Definition and evaluation of transient ischemic attack. Stroke. 2009;40(6):2276-2293. doi:10.1161/ strokeaha.108.192218

3. Edwards JD, Meehan SK, Levy AR, et al. Changes in intracortical excitability after transient ischemic attack are associated with ABCD2 score. Stroke. 2011;42(3):728-733. doi:10.1161/strokeaha.110.602938

4. Mahlknecht P, Kiechl S, Bloem BR, et al. Prevalence and burden of gait disorders in elderly men and women aged 60-97 years: a population-based study. PLoS One. 2013;8(7):e69627. doi:10.1371/ journal.pone. 0069627
5. Ortiz GA, Sacco RL. MD MS FAAN FAHA M. National Institutes of Health Stroke Scale (NIHSS). Wiley StatsRef: Statistics Reference Online; 2014; doi:10.1002/9781118445112.stat06823

6. Yang Y, Ding R, Hu D, et al. Reliability and validity of a Chinese version of the HADS for screening depression and anxiety in psycho-cardiological outpatients. Compr Psychiatry. 2014;55 (1):215-220. doi:10.1016/j.comppsych.2013.08.012

7. Whitney SL, Wrisley DM, Marchetti GF, et al. Clinical measurement of sit-to-stand performance in people with balance disorders: validity of data for the five-times-sit-to-stand test. Phys Ther. 2005;85(10):1034-1045.

8. Chan PP, Si Tou JI, Tse MM, et al. Reliability and validity of the timed up and go test with a motor task in people with chronic stroke. Arch Phys Med Rehabil. 2017;98(11):2213-2220. doi:10.1016/j. apmr.2017.03.008

9. Goh EY, Chua SY, Hong SJ, et al. Reliability and concurrent validity of Four Square Step Test scores in subjects with chronic stroke: a pilot study. Arch Phys Med Rehabil. 2013;94(7):1306-1311. doi:10.1016/j.apmr.2013.01.027

10. Alenazi AM, Alshehri MM, Alothman S, et al. Functional reach, depression scores, and number of medications are associated with number of falls in people with chronic stroke. Pmr. 2018;10 (8):806-816. doi:10.1016/j.pmrj.2017.12.005

11. Robinson RL, Ng SSM, Timed T. $180^{\circ}$ turn test for assessing people with hemiplegia from chronic stroke. Biomed Res Int. 2018;2018:9629230. doi:10.1155/2018/9629230

12. Trojian TH, McKeag DB. Single leg balance test to identify risk of ankle sprains. Br J Sports Med. 2006;40(7):610-613. doi:10.1136/ bjsm.2005.024356

13. Schneiders AG, Sullivan SJ, McCrory PR, et al. The effect of exercise on motor performance tasks used in the neurological assessment of sports-related concussion. $B r \quad J$ Sports Med. 2008;42 (12):1011-1013. doi:10.1136/bjsm.2007.041665

14. Gray VL, Goldberg AP, Rogers MW, et al. Asymptomatic carotid stenosis is associated with mobility and cognitive dysfunction and heightens falls in older adults. J Vasc Surg. 2020;71(6):1930-1937. doi:10.1016/j.jvs.2019.09.020

15. Rosenbaum Halevi D, Bursaw AW, Karamchandani RR, et al. Cognitive deficits in acute mild ischemic stroke and TIA and effects of rt-PA. Ann Clin Transl Neurol. 2019;6(3):466-474. doi:10.1002/acn3.719

16. Moran GM, Fletcher B, Feltham MG, et al. Fatigue, psychological and cognitive impairment following transient ischaemic attack and minor stroke: a systematic review. Eur J Neurol. 2014;21 (10):1258-1267. doi:10.1111/ene.12469

17. Israelsson H, Birgander R, Ambarki K, et al. Ventriculomegaly and balance disturbances in patients with TIA. Acta Neurol Scand. 2012;125(3):163-170. doi:10.1111/j.1600-0404.2011.01520.x

18. Batchelor FA, Williams SB, Wijeratne T, et al. Balance and Gait Impairment in Transient Ischemic Attack and Minor Stroke. J Stroke Cerebrovasc Dis. 2015;24(10):2291-2297. doi:10.1016/j. jstrokecerebrovasdis.2015.06.014

19. Piscitelli D, Falaki A, Solnik S, et al. Anticipatory postural adjustments and anticipatory synergy adjustments: preparing to a postural perturbation with predictable and unpredictable direction. Exp Brain Res. 2017;235(3):713-730. doi:10.1007/s00221-016-4835-x

20. Tjernström F, Fransson PA, Pate M, et al. Postural control and adaptation are influenced by preceding postural challenges. Exp Brain Res. 2010;202(3):613-621. doi:10.1007/s00221-010-2166-x

21. Oddsson LI. Control of voluntary trunk movements in man. Mech Postural Equilibrium During Standing Acta Physiol Scand Suppl. 1990;595:1-60.

22. Tinetti ME, Kumar C. The patient who falls: "It's always a trade-off. JAMA. 2010;303(3):258-266. doi:10.1001/jama.2009.2024

23. Navarro-Flores E, Romero-Morales C. Sex Differences in Frail Older Adults with Foot Pain in a Spanish Population: an Observational Study. Int $J$ Environ Res Public Health. 2020;17(17):6141. doi:10.3390/ijerph17176141 
24. Härlein J, Halfens RJ, Dassen T, et al. Falls in older hospital inpatients and the effect of cognitive impairment: a secondary analysis of prevalence studies. J Clin Nurs. 2011;20:175-183. doi:10.1111/ j.1365-2702.2010.03460.x

25. Patel P, Lamar M, Bhatt T. Effect of type of cognitive task and walking speed on cognitive-motor interference during dual-task walking. Neuroscience. 2014;260:140-148. doi:10.1016/j. neuroscience.2013.12.016

26. Ince PG, Minett $T$, Forster $G$, et al. Microinfarcts in an older population-representative brain donor cohort (MRC CFAS): prevalence, relation to dementia and mobility, and implications for the evaluation of cerebral Small Vessel Disease. Neuropathol Appl Neurobiol. 2017;43(5):409-418. doi:10.1111/nan.12363

27. Poredos P, Spirkoska A, Lezaic L, et al. Patients with an inflamed atherosclerotic plaque have increased levels of circulating inflammatory markers. J Atheroscler Thromb. 2017;24(1):39-46. doi:10.5551/jat.34884

28. Balestrini S, Perozzi C, Altamura C, et al. Severe carotid stenosis and impaired cerebral hemodynamics can influence cognitive deterioration. Neurology. 2013;80(23):2145-2150. doi:10.1212/ WNL.0b013e318295d71a

29. Duncombe J, Kitamura A, Hase Y, et al. Chronic cerebral hypoperfusion: a key mechanism leading to vascular cognitive impairment and dementia. Closing the translational gap between rodent models and human vascular cognitive impairment and dementia. Clin Sci. 2017;131(19):2451-2468. doi:10.1042/cs20160727

30. Jing Z, Shi C, Zhu L, et al. Chronic cerebral hypoperfusion induces vascular plasticity and hemodynamics but also neuronal degeneration and cognitive impairment. J Cerebral Blood Flow Metab. 2015;35 (8):1249-1259. doi:10.1038/jcbfm.2015.55

31. Wolters FJ, Zonneveld HI, Hofman A, et al. Cerebral Perfusion and the Risk of Dementia: a Population-Based Study. Circulation. 2017;136(8):719-728. doi:10.1161/circulationaha.117.027448

32. Bloch F, Thibaud M, Dugué B, et al. Psychotropic drugs and falls in the elderly people: updated literature review and meta-analysis. J Aging Health. 2011;23(2):329-346. doi:10.1177/0898264310381277

33. Woolcott JC, Richardson KJ, Wiens MO, et al. Meta-analysis of the impact of 9 medication classes on falls in elderly persons. Arch Intern Med. 2009;169(21):1952-1960. doi:10.1001/archinternmed.2009.357
34. Gillespie L, Robertson M, Gillespie W, et al. Interventions for preventing falls in older people living in the community. Cochrane Database Sys Rev. 2012;(9):CD007146. doi:10.1002/14651858. CD007146.pub3

35. Sherrington C, Fairhall NJ, Wallbank GK, et al. Exercise for preventing falls in older people living in the community. Cochrane Database Sys Rev. 2019;(1):CD012424. doi:10.1002/ 14651858.CD012424.pub2

36. Brognara N-F. Beneficial effect of foot plantar stimulation in gait parameters in individuals with Parkinson's disease. Brain Sci. 2020;10(2):69.

37. Hopewell S, Adedire O, Copsey BJ, et al. Multifactorial and multiple component interventions for preventing falls in older people living in the community. Cochrane Database Sys Rev. 2018;7:CD012221. doi:10.1002/14651858.CD012221.pub2

38. Gillespie LD, Gillespie WJ, Robertson MC, et al. WITHDRAWN: interventions for preventing falls in elderly people. Cochrane Database Sys Rev. 2009;(2):CD000340. doi:10.1002/14651858. CD000340.pub2

39. Shaw FE, Bond J, Richardson DA, et al. Multifactorial intervention after a fall in older people with cognitive impairment and dementia presenting to the accident and emergency department: randomised controlled trial. BMJ. 2003;326(7380):73. doi:10.1136/ bmj.326.7380.73

40. Andersen SD, Gorst-Rasmussen A, Lip GY, et al. Recurrent Stroke: the Value of the CHA2DS2VASc Score and the Essen Stroke Risk Score in a Nationwide Stroke Cohort. Stroke. 2015;46(9):2491-2497. doi:10.1161/strokeaha.115.009912

41. Pei L, Song B, Fang H, et al. Combining diffusion-weighted imaging patterns with ABCD2 score predicts stroke risk after transient ischemic attack. Curr Neurovasc Res. 2018;15(4):298-304. doi:10.2174/1567202616666181204121636

42. Wardlaw JM, Brazzelli M, Chappell FM, et al. ABCD2 score and secondary stroke prevention: meta-analysis and effect per 1000 patients triaged. Neurology. 2015;85(4):373-380. doi:10.1212/ wnl.0000000000001780
Neuropsychiatric Disease and Treatment

\section{Publish your work in this journal}

Neuropsychiatric Disease and Treatment is an international, peerreviewed journal of clinical therapeutics and pharmacology focusing on concise rapid reporting of clinical or pre-clinical studies on a range of neuropsychiatric and neurological disorders. This journal is indexed on PubMed Central, the 'PsycINFO' database and CAS, and

\section{Dovepress}

is the official journal of The International Neuropsychiatric Association (INA). The manuscript management system is completely online and includes a very quick and fair peer-review system, which is all easy to use. Visit http://www.dovepress.com/testimonials.php to read real quotes from published authors.

Submit your manuscript here: https://www.dovepress.com/neuropsychiatric-disease-and-treatment-journal 